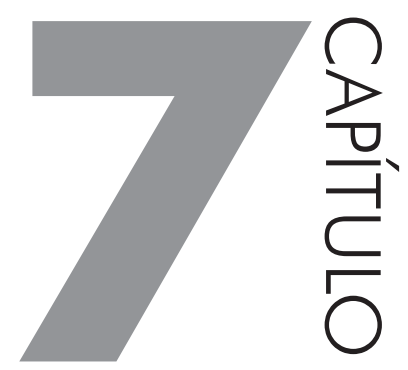

\title{
ESTUDO EXPERIMENTAL E NUMÉRICO SOBRE REMOÇÃO DE SEDIMENTOS NUM TANQUE DE LASTRO
}

\section{LIANG-YEE CHENG, PEDRO HENRIQUE SAGGIORATTO OSELLO, LUCAS SOARES PEREIRA, GABRIEL HENRIQUE DE SOUZA RIBEIRO, FABIO KENJI MOTEZUKI}

\subsection{INTRODUÇÃO}

O transporte de água de lastro a longas distâncias pelas embarcações e seu despejo no destino são fontes de preocupação por causa do impacto ambiental que pode ser gerado pela introdução de espécies e/ou materiais não nativos. Como medida de controle, diversas técnicas de monitoração e tratamento de água de lastro têm sido propostas. Uma das abordagens são as técnicas de renovação da água de lastro durante a viagem, tais como flow-through ou sequential exchange.

Nos últimos anos, diversos estudos sobre a eficácia destas técnicas foram realizados focando essencialmente na qualidade da água de lastro e segurança operacional. No entanto, a presença dos sedimentos, que apresentam propriedades distintas da água, é sistematicamente desprezada. No caso de tanques de duplo fundo das embarcações, que normalmente são utilizados como tanques para água de lastro, os sedimentos depositados e carregados à longa distância são agravantes do impacto ambiental, porque podem propiciar ambientes favoráveis para a proliferação de micro-organismos. 
Remoção de sedimentos é um processo importante em diversas áreas de engenharia e um fenômeno extremamente complexo devido ao grande número de fatores intervenientes e neste trabalho o processo de remoção de sedimento nos tanques de duplo fundo é investigado por meio de abordagens experimental e numérica.

Por simplicidade, experimentos físicos foram conduzidos, onde o sedimento é aproximado por pequenas esferas de material homogêneo. Numericamente, tendo em vistas as propriedades distintas entre a água e o sedimento, que é essencialmente uma camada de material lamacento, ambos foram modelados como fluídos homogêneos, newtonianos, imiscíveis e de densidades diferentes. A fim de capturar melhor a complexa interface entre os fluídos, o método de simulação Moving Particle Semi-Implicit (MPS), que é baseado em descrição lagrangeana e discretização do meio contínuo em partículas foi adotado. Por meio da análise comparativa entre os resultados numéricos e experimentais, e levando em conta as semelhanças e diferenças, entre os dois, são discutidos os mecanismos da remoção do sedimento, assim como os méritos e deficiências do modelo numérico.

\subsection{MODELO EXPERIMENTAL}

O experimento foi concebido baseado em modelos de tanque de lastro de duplo fundo com duto conectado a uma bomba para promover a circulação de água. Apesar do procedimento de troca de água do lastro ser realizado num ambiente dinâmico, com excitação provocada pelas ondas, o ensaio será realizado considerando condições estáticas, objetivando facilitar a análise do fenômeno.

\subsubsection{Dimensões do modelo experimental}

A Figura 36 apresenta um exemplo da configuração estrutural do tanque de lastro de um navio graneleiro. O espaço onde é armazenada a água de lastro do navio se encontra entre o chapeamento do fundo do porão de carga e o chapeamento do fundo do casco, e por isso é conhecido como duplo fundo. Nessa região estão localizados diversos elementos estruturantes da embarcação, além disso é compartimentada por anteparas estanques ou comunicantes, resultando em diversos tanques de geometria similar. 


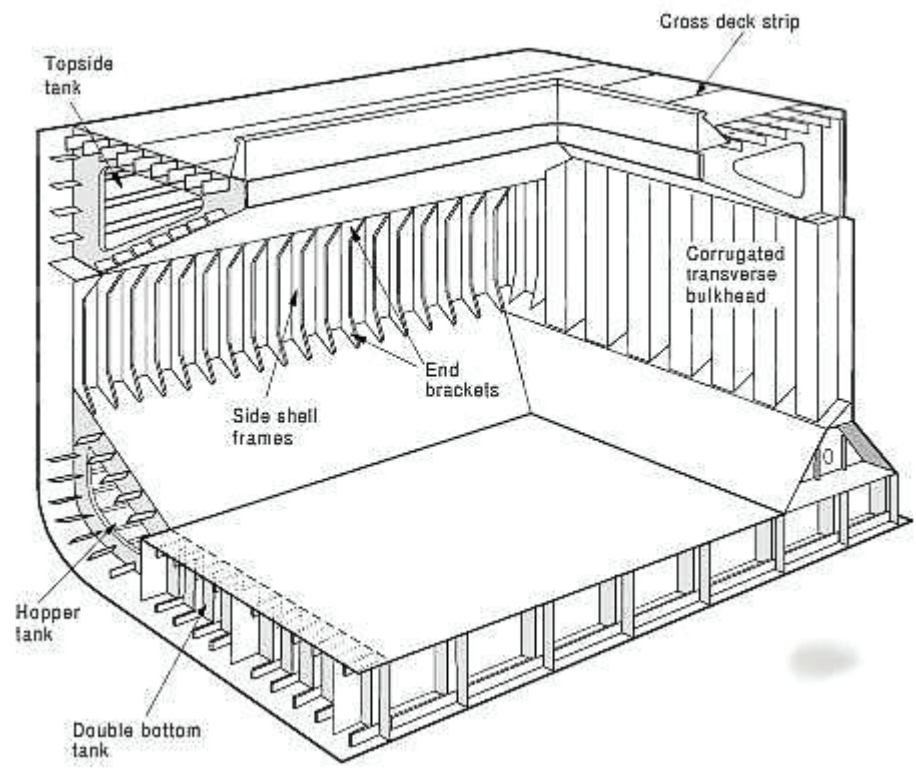

Figura 36 Perspectiva em corte do porão de uma embarcação com tanque de duplo fundo

O modelo experimental projetado é uma representação em escala reduzida de um tanque de duplo fundo, ao considerar as dimensões típicas do tanque e o espaço disponível no laboratório foi adotada a escala de 1:4. A concepção do modelo foi realizada de forma a tornar o experimento flexível, possibilitando diversas configurações e reutilização das peças. De maneira geral o modelo projetado é constituído por um tanque externo resistente e um gabarito interno adaptável, possibilitando a modificação da configuração interna do tanque sem a necessidade de construção de um novo.

Além de modelar a configuração normal do tanque de lastro, o modelo utilizado nesse trabalho também foi projetado para investigar a influência da instalação de defletor e sua inclinação (ângulo de ataque) no direcionamento do escoamento dentro do tanque e a sua eficácia na remoção dos sedimentos durante a operação de troca da água de lastro. Por simplicidade, o modelo experimental apresentado será o relativo às simulações $2 \mathrm{D}$, como pode ser visto na Figura 37. A espessura do tanque é de $5,0 \mathrm{~cm}$, desconsiderando a espessura das placas. 

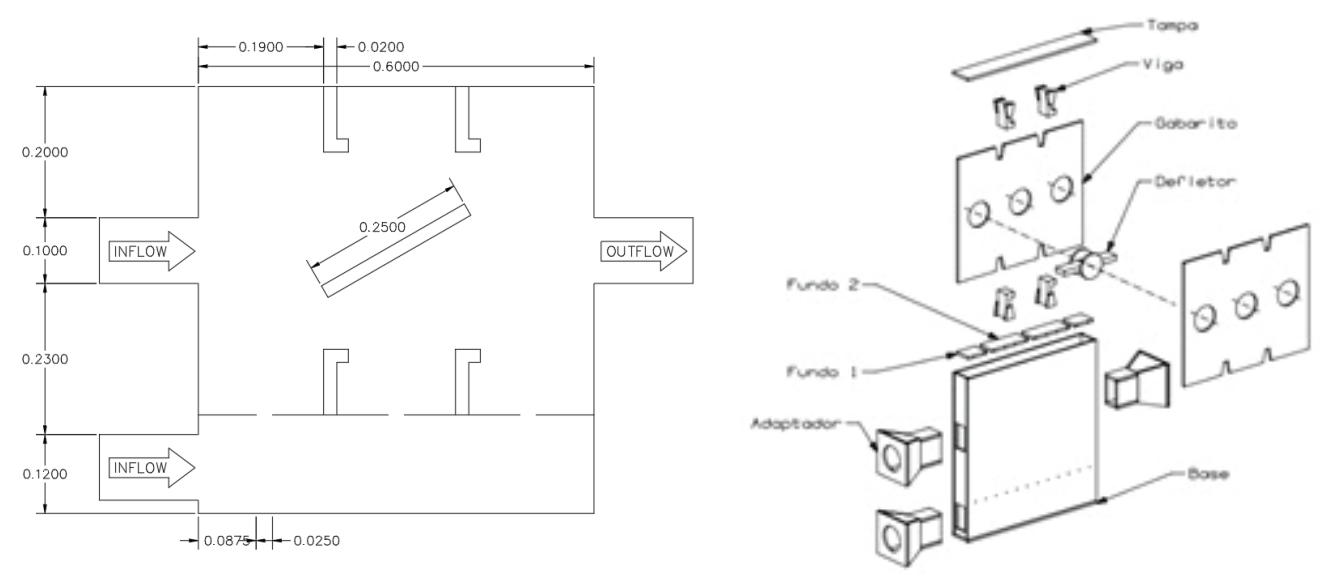

Figura 37 Seção longitudinal média do tanque (esquerda) e visualização explodida do modelo laboratorial (direita). 0 duto de saída da parte inferior do tanque do tanque (abaixo da linha pontilhada) não foi usado nesta fase inicial dos estudos

\subsubsection{Conjunto Experimental}

O conjunto experimental completo abrange o sistema de armazenamento da água, os dispositivos de medição, o mecanismo de controle de vazão, o sistema de recuperação da água utilizada nos ensaios e o dispositivo para realização do recalque.

As caixas d'águas responsáveis por fornecer a água para o tanque estão localizadas em pavimento superior ao modelo, esse desnível é responsável pela circulação da água durante os ensaios. O desnível entre os pavimentos é de cerca de 2,7 $\mathrm{m}$. O sistema instalado no pavimento superior consiste de quatro caixas interconectadas por tubulações. No barrilete superior estão instalados dois dispositivos de perda de carga localizados, que possibilitam a medição da vazão.

Após a passagem pelos dispositivos de perda de carga, o fluído segue pelo tubo de queda vertical, onde está localizado o registro gaveta, que é responsável pelo controle de vazão durante os ensaios. Depois da curva da queda vertical o fluído entra no modelo, que já foi visto em detalhes na subsecção anterior. No tanque estão instalados sensores de pressão, que juntamente com os sensores de pressão do barrilete superior formam o painel de manômetros.

Depois de passar pelo modelo, a água segue pela tubulação até alcançar o reservatório inferior. No fim dessa tubulação foi instalada uma rede para a separação do material utilizado como sedimento durante os ensaios, de forma a evitar que o material pudesse ser succionado pela bomba de recalque. O reservatório inferior é formado por um conjunto de quatro caixas d'água, que são conectadas de forma a distribuir o volume de água proveniente dos ensaios. 
O conjunto experimental fica completo com a utilização de bomba e mangueira, que é responsável pelo recalque do fluído utilizado durante os ensaios. A bomba é posicionada dentro do reservatório inferior, enquanto a saída fica no reservatório superior.

A Figura 38 apresenta uma visão esquemática do conjunto completo do experimento.

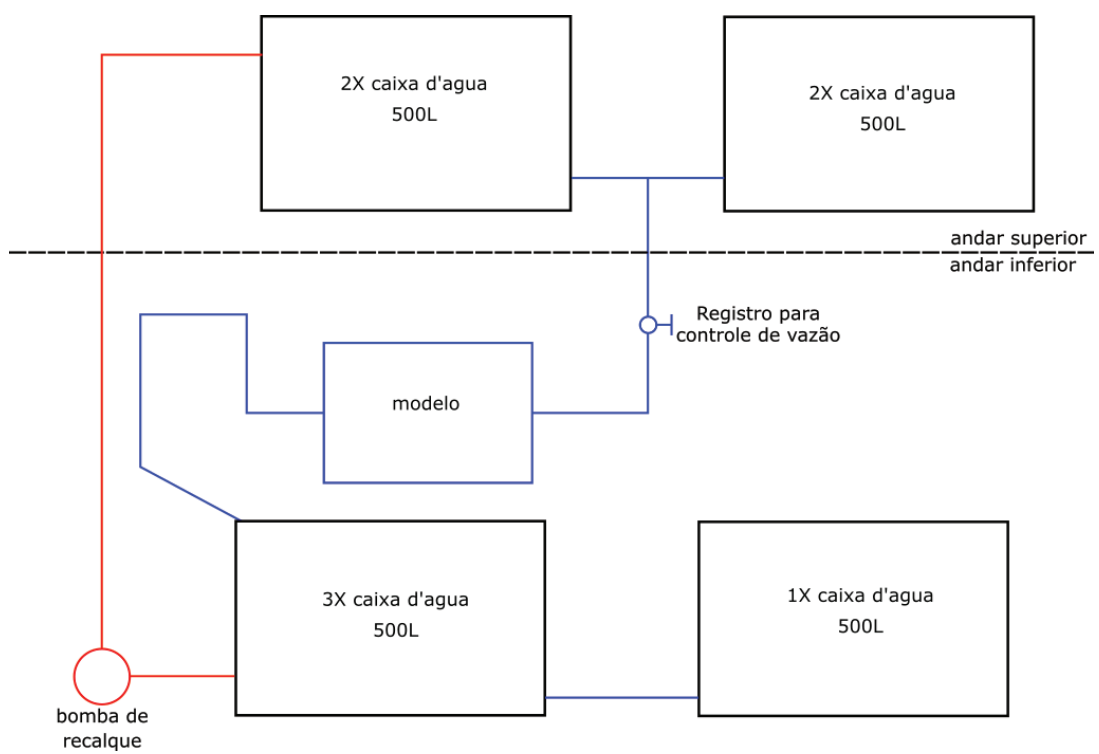

Figura 38 Esquema completo do conjunto do experimento de tanque de lastro

\subsubsection{Calibração dos manômetros}

Os sensores de pressão instalados antes e depois da perda de carga localizada no barrilete superior foram projetados especificamente para que fosse possível a medição da vazão durante os ensaios. A correlação entre a perda de carga medida e a velocidade do escoamento é realizada a partir da seguinte formulação:

$$
h_{f}=K \frac{v^{2}}{2 g}
$$

Sendo $h_{f}$ a perda de carga localizada, $K$ o coeficiente relativo do dispositivo em uso, $g$ a aceleração da gravidade e $v$ a velocidade do fluxo. Portanto, a correlação entre perda de carga localizada medida nos manômetros e a velocidade do fluxo depende apenas do coeficiente $K$, que pode ser determinado experimentalmente. A determinação desse valor dá-se o nome de calibração do manômetro e foi realizada experimentalmente a partir da medição da vazão de saída do conjunto de acordo com diversas aberturas do registro gaveta. A medição da vazão 
de saída foi realizada a partir da medição do tempo necessário para encher um balde de 22,45 l. Enquanto isso, foi realizada a filmagem da variação do nível dos manômetros. As Figura 39 e Figura 40 mostram a relação dos valores obtidos para os dispositivos de perda de carga localizada:

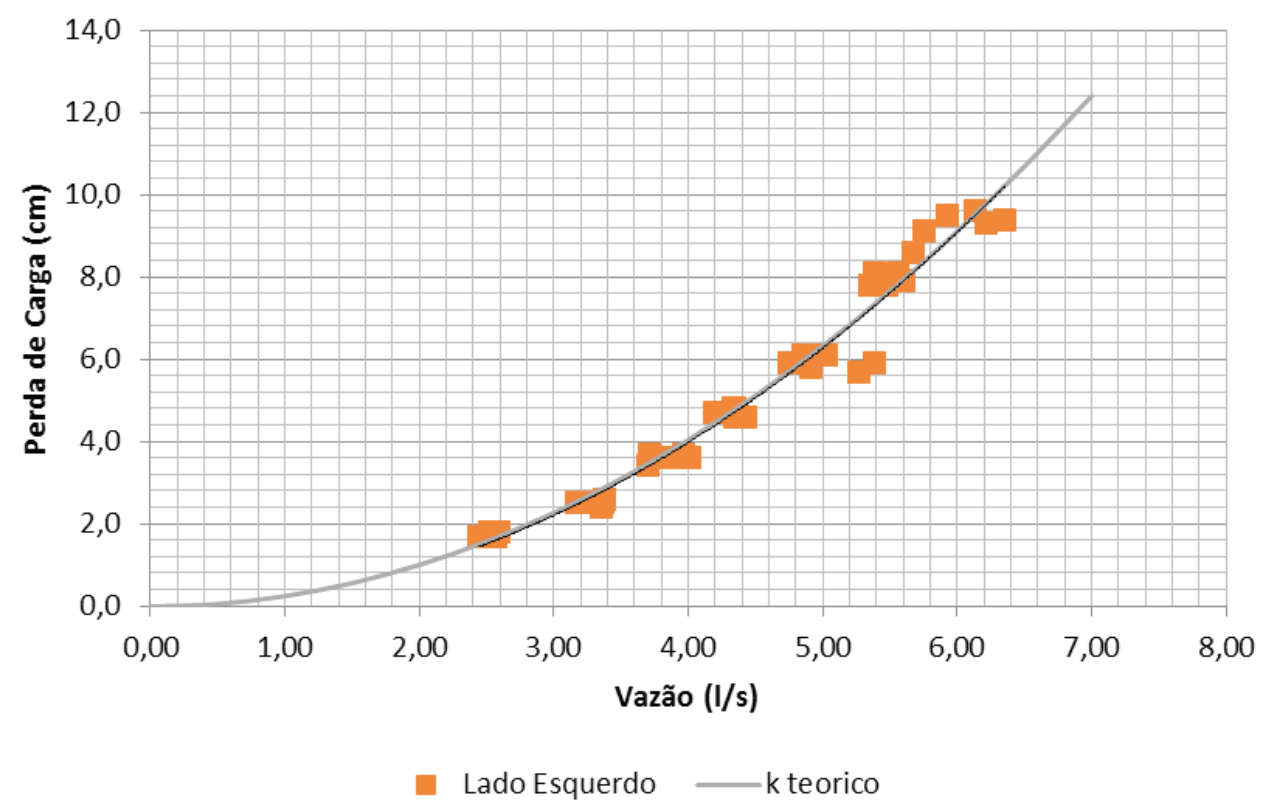

Figura 39 Curva de calibração do manômetro 1

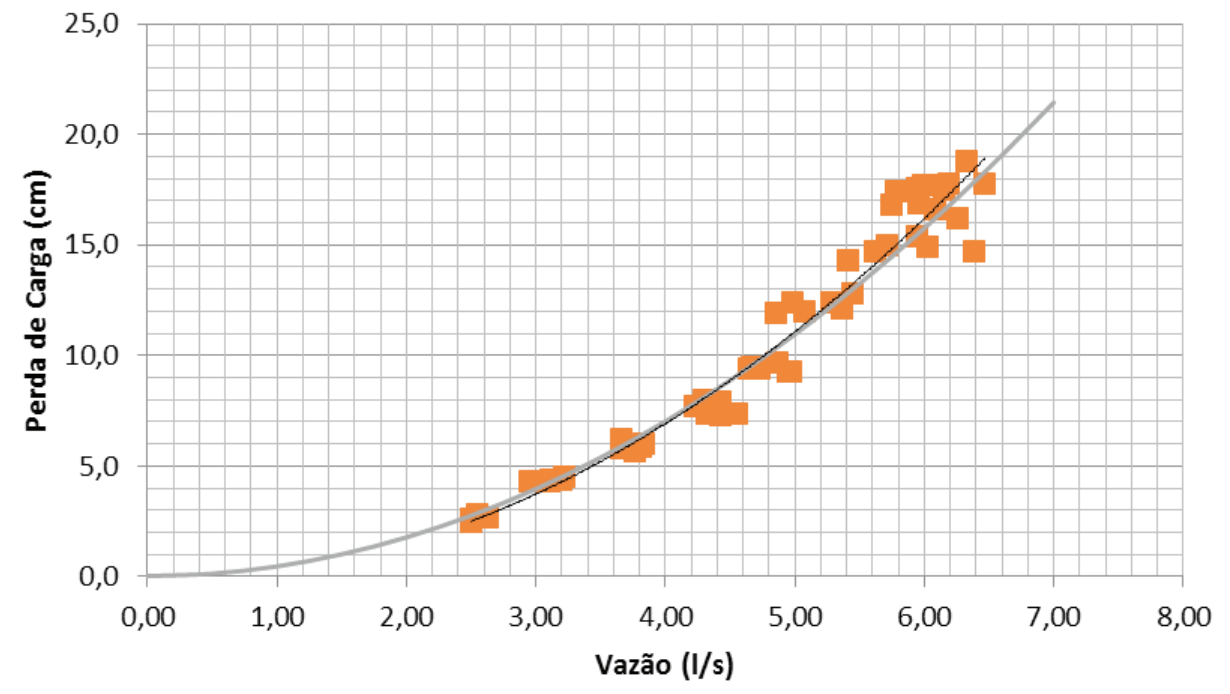

- Manômetro 2 - kteorico

Figura 40 Curva de calibração do manômetro 2 
A curva do $K$ teórico foi obtida a partir da média dos valores de cada ponto, calculados a partir da Equação 1. A proximidade entre essa curva e a linha de tendência dos pontos demonstra a consistência do método empregado. Para o manômetro 1, o valor de $K$ é igual a 0,968 e para o manômetro 2, o valor de $K$ é igual a 1,676 .

\subsection{MÉTODO NUMÉRICO}

\subsubsection{Equações governantes}

As equações governantes para o escoamento incompressível são as equações da conservação de massa e do momento, Eqs. (2) e (3):

$$
\begin{gathered}
\frac{\mathrm{D} \rho}{\mathrm{D} t}+\rho \nabla \cdot \overrightarrow{\mathrm{v}}=0 \\
\frac{\mathrm{D} \vec{v}}{\mathrm{D} t}=-\frac{1}{\rho} \nabla P+v \nabla^{2} \vec{v}+\vec{f}
\end{gathered}
$$

onde $\rho$ é a massa específica, $t$ o tempo, $\vec{v}$ a velocidade, $P$ a pressão, $v$ a viscosidade cinemática e $\vec{f}$ a força externa.

\subsubsection{Operadores diferenciais discretos}

No método MPS, todos os termos das equações governantes, representados por operadores diferenciais, são substituídos por operadores diferenciais discretos em uma distribuição irregular de nós, onde, para cada partícula $i$, a influência de uma partícula vizinha $j$ é definida pela função peso $\omega_{\mathrm{ij}}$ :

$$
\omega_{i j}=\left\{\begin{array}{cc}
\frac{r_{e}}{\left\|\vec{r}_{i j}\right\|}-1 & \left\|\vec{r}_{i j}\right\| \leq r_{e} \\
0 & \left\|\vec{r}_{i j}\right\|>r_{e}
\end{array}\right.
$$

onde $r_{\mathrm{e}}$ é o raio efetivo, que estabelece quais partículas $j$ devem ser consideradas no cálculo das grandezas físicas da partícula $i$, e $\left\|\vec{r}_{i j}\right\|$ é a distância entre as partículas $i$ e $j$.

Para cada partícula $i$, a somatória dos pesos de todas as partículas vizinhas $j$, é definido como a densidade do número de partículas $n_{\mathrm{i}}$ :

$$
n_{i}=\sum_{j \neq i} \omega_{i j}
$$

Desta forma, para uma função escalar $\varphi$, os operadores gradiente e laplaciano são definidos pelas Eqs. (6) e (7), respectivamente: 


$$
\begin{aligned}
\langle\nabla \varphi\rangle_{i} & =\frac{d}{n_{\max }^{0}} \sum_{j \neq i} \frac{\left(\varphi_{j}-\varphi_{i}\right)}{\left\|\vec{r}_{i j}\right\|^{2}} \vec{r}_{i j} \omega_{i j} \\
\left\langle\nabla^{2} \varphi\right\rangle_{i} & =\frac{2 d}{\lambda n_{\max }^{0}} \sum_{j \neq i}\left(\varphi_{j}-\varphi_{i}\right) \omega_{i j}
\end{aligned}
$$

onde $d$ é a dimensão espacial, $n_{\max }^{0}$ é o valor inicial máximo de $n_{\mathrm{i}}$ e $\lambda$ é um parâmetro de correção, que faz o ajuste do crescimento da variância e pode ser calculado como:

$$
\lambda=\frac{\sum_{j \neq i}\left\|\vec{r}_{i j}\right\|^{2} \omega_{i j}}{\sum_{j \neq i} \omega_{i j}}
$$

Para o escoamento incompressível, um algoritmo semi-implícito é utilizado no método MPS. Inicialmente, velocidade e posição são calculadas explicitamente, considerando os termos de viscosidade e força externa da equação de conservação do momento, Eq. (3). Após os cálculos da parte explícita, a pressão de todas as partículas é calculada implicitamente pela solução do sistema linear formado pela equação de Poisson para a pressão:

$$
\left\langle\nabla^{2} P\right\rangle_{i}^{t+\Delta t}-\alpha \frac{\rho}{\Delta t^{2}} P_{i}^{t+\Delta t}=-\gamma \frac{\rho}{\Delta t^{2}} \frac{n_{i}^{*}-n_{\max }^{0}}{n_{\max }^{0}}
$$

onde $n_{\mathrm{i}}{ }^{*}$ é a densidade do número de partículas calculada após os deslocamentos das partículas na fase explícita, $\alpha$ é o coeficiente de compressibilidade artificial que permite uma compressibilidade limitada e acelera a convergência da solução do sistema linear e $\gamma$ é o coeficiente de relaxação que é utilizado para reduzir oscilações de pressão artificiais. Além disso, o gradiente de pressão também é modificado para melhora da estabilidade do MPS (KOSHIZUKA et al., 1996):

$$
\langle\nabla P\rangle_{i}=\frac{d}{n_{\max }^{0}} \sum_{j \neq i} \frac{\left(\mathrm{P}_{j}-\hat{\mathrm{P}}_{i}\right)}{\left\|\vec{r}_{i j}\right\|^{2}} \vec{r}_{i j} \omega_{i j}
$$

onde $\widehat{P}_{i}$ é a pressão mínima entre os vizinhos da partícula $i$, garantindo exclusivamente forças de repulsão, evitando sobreposição entre partículas.

Por fim, a velocidade das partículas é atualizada, considerando somente o termo do gradiente de pressão, e a nova posição das partículas é obtida. 


\subsection{CONDIÇÕES DE CONTORNO}

\subsubsection{Superfície livre}

A identificação das partículas de superfície livre é feita com base no número de densidade de partículas. Uma partícula é definida como partícula de superfície livre e sua pressão igualada à zero, quando seu número de densidade de partículas $n_{\mathrm{i}}$ é menor do que $\beta n_{\mathrm{i}}$, com $0<\beta<1$.

\subsubsection{Paredes rígidas}

As paredes sólidas são representadas por três camadas de partículas fixas. As partículas em contato com fluido são denominadas partículas de parede, com suas pressões calculadas pela equação de Poisson para a pressão, Eq. (9), juntamente com as partículas de fluido. As partículas que formam as outras duas camadas são denominadas partículas dummy. As partículas dummy são utilizadas para garantir o cálculo correto do número de densidade de partículas nas partículas de parede, não sendo calculada a pressão destas partículas.

\subsubsection{Contorno com recirculação}

Para a modelagem das condições de entrada e saída de fluxo no tanque, foi utilizada a condição de contorno de recirculação proposta por BELLEZI et al. (2013). A condição de contorno essencialmente tenta reproduzir um escoamento em circuito fechado, melhorando a eficiência computacional e reduzindo o domínio de todo circuito por meio da adoção de condição de periodicidade associada a uma região de transição, onde as velocidades são ajustadas de forma que o fluído é reintroduzido no domínio computacional segundo um perfil desejado. A esta região de ajuste convencionou-se chamar de controlador de fluxo.

O funcionamento do controlador de fluxo é dividido em dois estágios sucessivos ao longo do tempo. O primeiro estágio, em regime transiente, aplica um campo de acelerações horizontal para iniciar o escoamento das partículas em repouso. A duração desta etapa, definida como $t_{\text {ramp }}$, é determinada com base no tempo necessário para uma partícula em repouso atingir a velocidade desejada para a injeção. Passado este período de tempo dá-se início ao segundo estágio, em regime permanente. No segundo estágio o ajuste para a circulação do fluído ocorre exclusivamente na região do controlador de fluxo, definida no início da simulação como um dos parâmetros de entrada necessários, o controlador de fluxo compreende um trecho na entrada da geometria e um trecho na saída. Considerando o fluxo na direção horizontal, dentro do controlador, a velocidade ho- 
rizontal das partículas é ajustada para a velocidade desejada e a velocidade vertical suprimida. O controle da velocidade é realizado por meio da adição de uma componente de velocidade horizontal durante a etapa explícita de cálculo.

A aceleração é imposta adicionando-se um termo à equação de conservação de momento resolvida durante o passo explícito do algoritmo do MPS aplicado às partículas dentro do volume de controle conforme mostrado na Eq. 11, onde $\rho$ é a densidade do fluido, $u$ a velocidade, $g$ a aceleração da gravidade, $f$ as forças externas, $v$ a viscosidade cinemática e $t$ o tempo.

$$
\left[\frac{D \vec{u}}{D t}\right]_{\text {explicito }}=v \nabla^{2} \vec{u}+\frac{f}{\rho}+\vec{g}+\frac{\partial \vec{u}_{f l u x o}}{\partial t}
$$

O termo $\frac{\partial \vec{u}_{f l u x o}}{\partial t}$ é calculado comparando-se a velocidade atual de cada partícula do fluido dentro do volume de controle e aplicando uma aceleração proporcional à diferença entre a velocidade atual da partícula e a velocidade desejada $U$. A aceleração é ainda ponderada por um fator de suavização do tipo senoidal para evitar descontinuidade no domínio computacional. Dessa forma o termo pode ser calculado conforme mostrado na Eq. 12, onde $x_{0}$ e $x_{\mathrm{f}}$ são os limites horizontais do volume de controle e $a_{1}$ uma calibração da intensidade de aceleração.

$$
\frac{\partial \vec{u}_{f u x}}{\partial t}=(U-u) \cdot a_{1} \frac{\sin \left(\pi \frac{x-x_{o}}{x_{f}-x_{f}}-\frac{\pi}{2}\right)+1}{2}
$$

Por fim, para evitar que as partículas de sedimento saindo da simulação sejam reintroduzidas, um filtro de partículas foi implementado junto ao controlador de fluxo, de modo que na saída da geometria, é feita uma verificação adicional do tipo de material, e caso não corresponda ao material do fluido, suas propriedades como densidade e viscosidade, bem como o identificador de tipo de material, são alteradas (BELLEZI et al., 2013).

\subsection{CASOS ESTUDADOS}

No caso do fenômeno estudado, por se tratar do escoamento por uma sequência de compartimentos idênticos, uma forma utilizada para reduzir o domínio experimental e o da computação sem comprometer a qualidade dos resultados é focar a análise do fenômeno de forma localizada, individualmente em um dos compartimentos. Adicionalmente, por simplicidade, foi utilizada uma geometria bidimensional, correspondente à seção longitudinal média do compartimento. Nos experimentos físicos, para aproximar o comportamento bidimensional, a 
espessura interna do tanque modelo é de $5 \mathrm{~cm}$. A seção longitudinal do tanque tem formato retangular, com 0,5 $m$ de altura e 0,6 $m$ de comprimento, ambos os dutos de entrada e saída do escoamento têm $0,1 m$ de altura e alinhadas horizontalmente. No interior do tanque existem 4 componentes estruturais na forma de vigas "L", dois na parte superior do tanque e dois na parte inferior. Adicionalmente, como uma possível medida de potencializar a remoção do sedimento, um defletor de 0,25 $m$ com inclinação ajustável foi instalado no centro do tanque. Uma representação da geometria é apresentada na Figura 37, junto a uma visualização explodida do modelo físico usado em laboratório.

Com a finalidade de verificar a efetividade do método computacional, tanto simulações como experimentos físicos foram realizados. Para isso, 8 casos foram escolhidos, levando em consideração duas vazões de bombeamento diferentes, correspondentes às velocidades de entrada de $0,45 \mathrm{~m} / \mathrm{s}$ e $0,9 \mathrm{~m} / \mathrm{s}$, duas densidades de sedimentos diferentes, sendo $1040 \mathrm{~kg} / \mathrm{m}^{3}$, correspondente às esferas de ABS com diâmetro médio de $5 \mathrm{~mm}$; e $2400 \mathrm{~kg} / \mathrm{m}^{3}$, correspondente às esferas de vidro com diâmetro médio de $2 \mathrm{~mm}$, e duas geometrias distintas: uma contendo apenas as vigas estruturais e uma com a adição de um defletor de fluxo, com o intuito de melhorar a eficiência na remoção de sedimentos. Para os casos aqui apresentados, a inclinação do defletor usada foi de $45^{\circ}$ no sentido horário. Para facilitar a compreensão, os casos estudados serão referidos de acordo com a nomenclatura fornecida na Tabela 9.

Tabela 9 Casos estudados

\begin{tabular}{|c|c|c|c|c|c|}
\hline NOME & SEDIMENTo & $\begin{array}{c}\text { DENSIDADE } \\
\left(\text { KG/M } \mathbf{M}^{\mathbf{3}}\right.\end{array}$ & DEFLETOR & $\begin{array}{c}\text { ÂNGULO Do } \\
\text { DEFLETOR ( })\end{array}$ & $\begin{array}{c}\text { VELOCIDADE } \\
\text { (M/S) }\end{array}$ \\
\hline AS1 & ABS & 1040 & Não & - & 0,45 \\
\hline AS2 & ABS & 1040 & Não & - & 0,90 \\
\hline AC1 & ABS & 1040 & Sim & 45 & 0,45 \\
\hline AC2 & ABS & 1040 & Sim & 45 & 0,90 \\
\hline MS1 & Vidro & 2400 & Não & - & 0,45 \\
\hline MS2 & Vidro & 2400 & Não & - & 0,90 \\
\hline MC1 & Vidro & 2400 & Sim & 45 & 0,45 \\
\hline MC2 & Vidro & 2400 & Sim & 45 & 0,90 \\
\hline
\end{tabular}

\subsubsection{Parâmetros computacionais}

Os 8 casos foram modelados com distância inicial entre partículas, $l_{0}$, de $0,002 \mathrm{~m}$, resultando em geometrias com cerca de 100 mil partículas. Foi usado 
um passo de tempo de $10^{-4}$. O coeficiente de relaxação $\gamma$ usado foi de 0,003 e o coeficiente de compressibilidade artificial $\alpha$ de $2,0.10^{-8}$, estes valores foram obtidos empiricamente de modo que as oscilações de pressão e o número de iterações para a convergência do sistema linear fossem minimizados enquanto o escoamento continua sendo considerado incompressível.

Em todos os casos o intervalo de estudo do fenômeno foi de $30 \mathrm{~s}$, com dois segundos adicionais utilizados para acelerar o escoamento até a velocidade desejada e estabilizá-lo pela condição de recirculação. Além disso, para levar em consideração a água presente nos interstícios do sedimento no experimento laboratorial, foi utilizada a densidade submersa do sedimento, de modo que para o ABS a densidade utilizada foi de $1020 \mathrm{~kg} / \mathrm{m}^{3}$ e para o vidro $1700 \mathrm{~kg} / \mathrm{m}^{3}$. Ambas as densidades foram determinadas em laboratório.

A plataforma computacional utilizada possui dois processadores Intel ${ }^{\circledR}$ Xeon ${ }^{\circledR}$ E5-2680@2.80GHz com 10 núcleos de processamento cada e $125 \mathrm{~Gb}$ de memória RAM. Neste equipamento, utilizando o modelo do tanque de lastro com 32s de simulação, são consumidos por volta de 30 horas de processamento.

\subsection{RESULTADOS}

A análise dos resultados será baseada na comparação de imagem em três instantes. O primeiro instante se refere ao momento de estabilização da velocidade do fluído dentro do tanque, ou seja, instante em que o fenômeno está em regime permanente, que no modelo numérico corresponde ao instante de alcance da velocidade final pelo controlador de fluxo e no modelo experimental ao instante de estabilização dos manômetros de pressão relativos ao tanque. O segundo instante será considerado o tempo necessário para a circulação de uma quantia de fluído correspondente a três vezes o volume do tanque a partir do instante de estabilização da velocidade. $\mathrm{O}$ terceiro instante será relativo ao momento de término de remoção de sedimentos do tanque.

Os resultados obtidos para seis dos casos estudados (AS1, AS2, AC1, AC2, MS2 e MC2) serão discutidos a seguir das Figura 41 - Figura 46. 


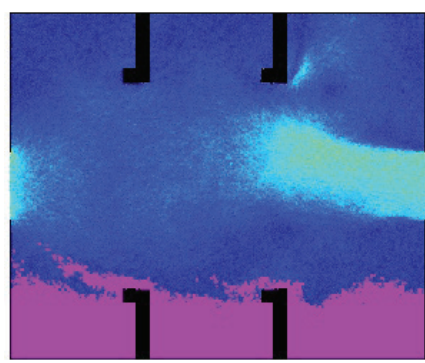

(a)

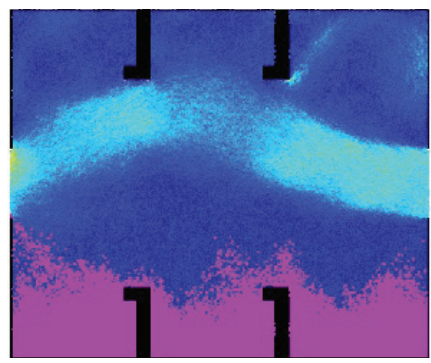

(d)

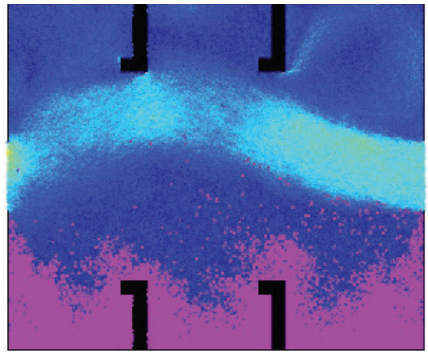

(g)

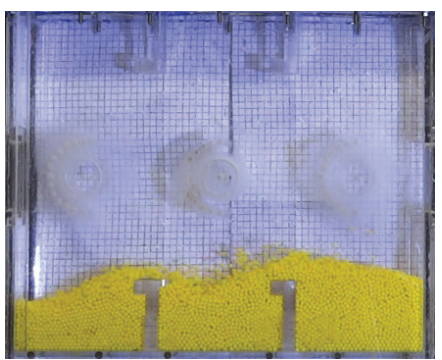

(b)

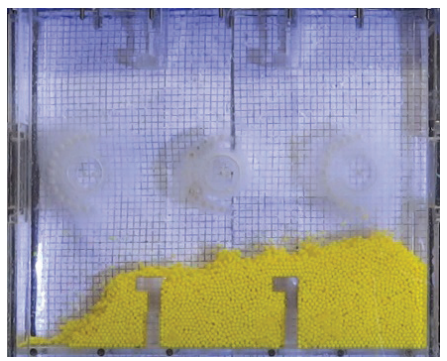

(e)

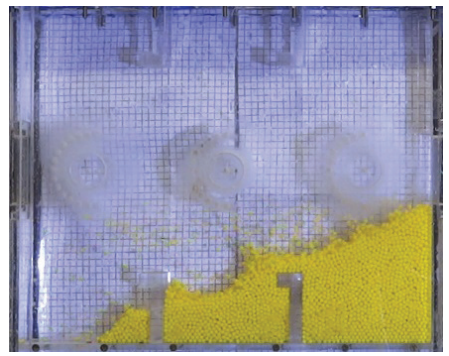

(h)

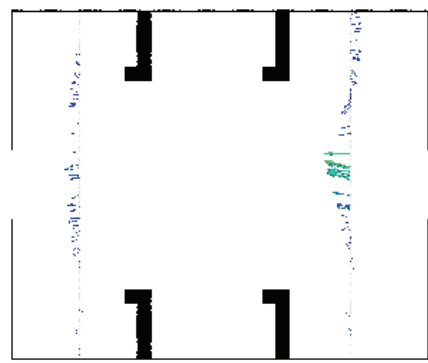

(c)

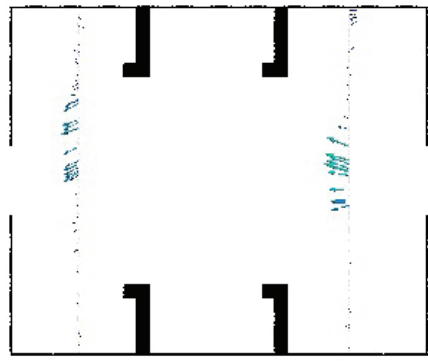

(f)

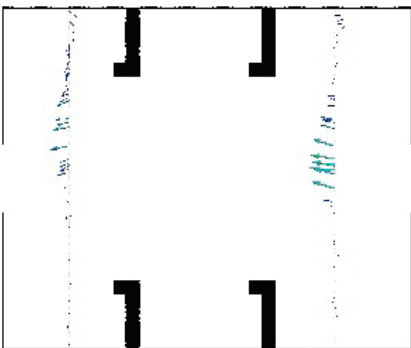

(i)

Velocity $(\mathrm{m} / \mathrm{s})$

0.0

0.3

0.6

0.9 1.2

Figura 41 Comparação dos resultados dos casos AS1: resultado numéricos (a, d, g), resultados experimentais $(b, e, h)$, perfis de velocidade $(c, f, i)$, primeiro instante $(a-c)$, segundo instante $(d-f)$ e terceiro instante $(g-i)$ 


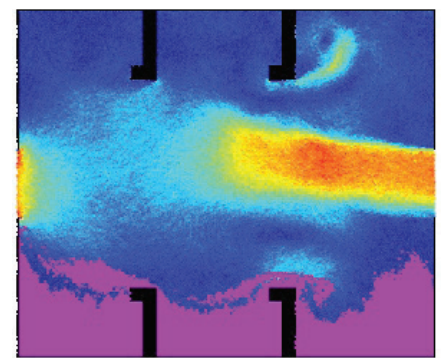

(a)

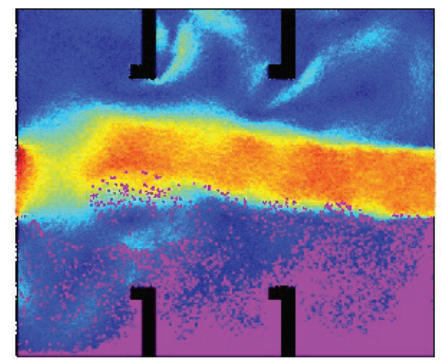

(d)

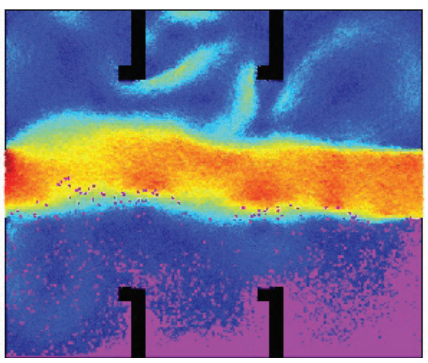

(g)

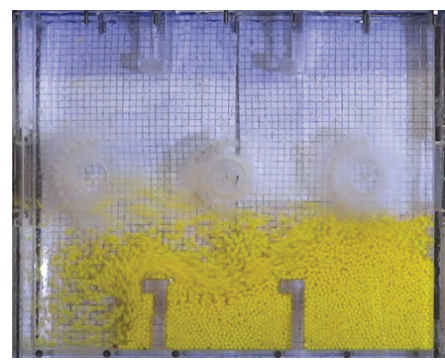

(b)

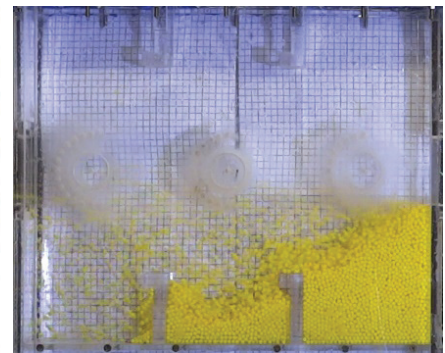

(e)

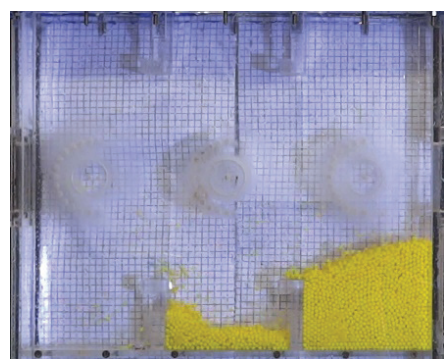

(h)

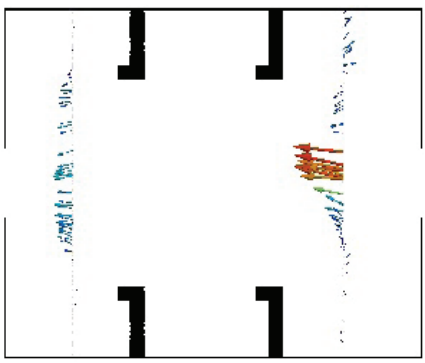

(c)

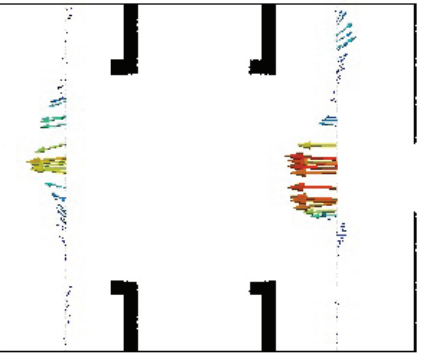

(f)

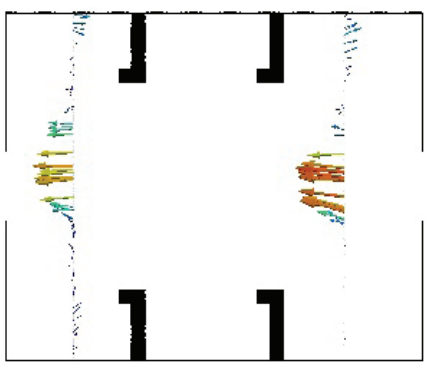

(i)

Velocity (m/s)

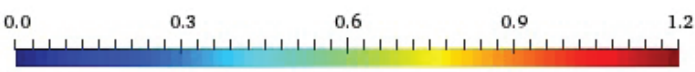

Figura 42 Comparação dos resultados dos casos AS2: resultado numéricos (a, d, g), resultados experimentais $(b, e, h)$, perfis de velocidade $(c, f, i)$, primeiro instante $(a-c)$, segundo instante $(d-f)$ e terceiro instante $(g-i)$

Comparando os casos AS1 e AS2, Figs. 41 e 42 respectivamente, devido a maior vazão o caso AS2 apresenta formação de vórtices mais intensa enquanto que no caso AS1 os vórtices são menores e concentrados próximos ao centro do tanque, como mostram os resultados numéricos e observado na baixa remoção de sedimento no experimento. 
O caso AS2 também mostra nos resultados experimentais uma maior suspensão e remoção de sedimentos comparado ao caso AS1, este comportamento também é observado nos resultados numéricos que mostram uma boa aproximação com o experimento no caso AS2.

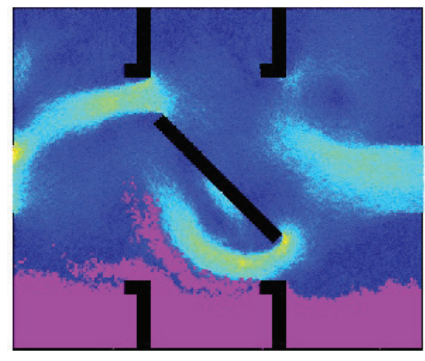

(a)

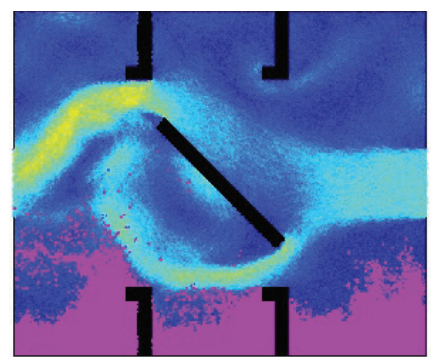

(d)

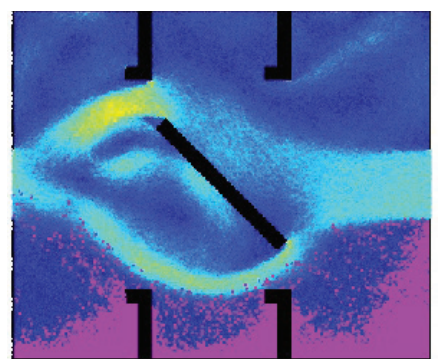

(g)

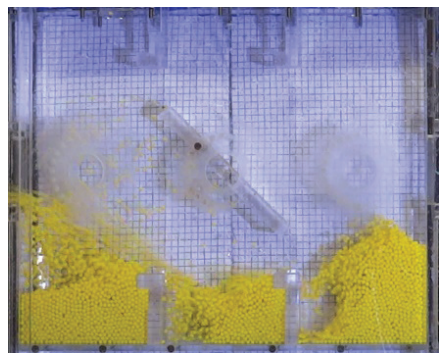

(b)

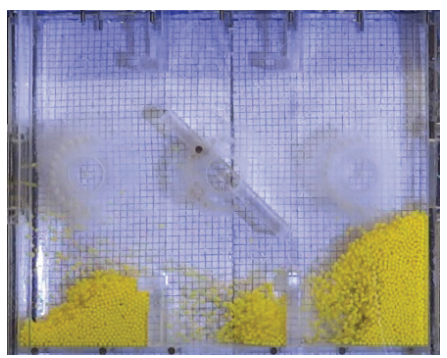

(e)

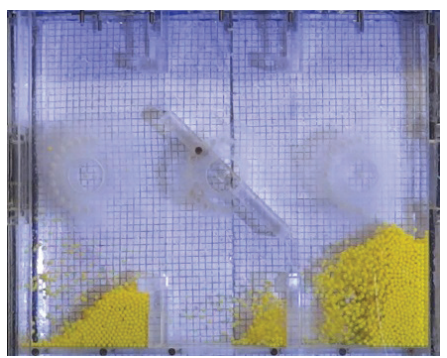

(h)

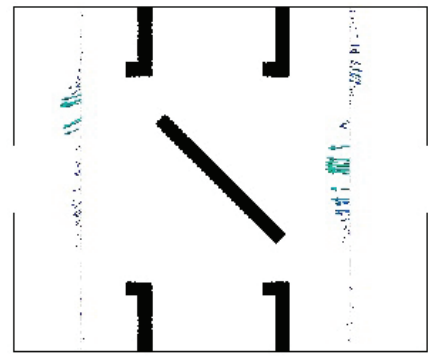

(c)

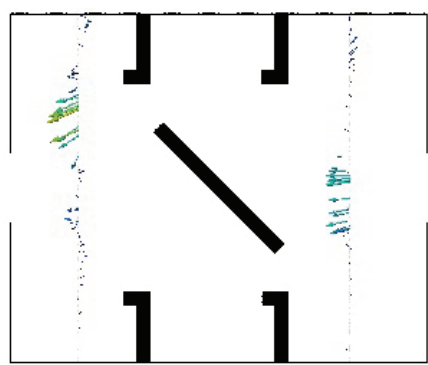

(f)

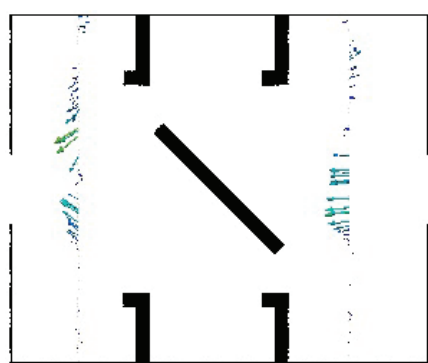

(i)

Velocity $(\mathrm{m} / \mathrm{s})$

0.0

0.3

0.6

0.9

1.2

Figura 43 Comparação dos resultados dos casos AC1: resultado numéricos (a, d, g), resultados experimentais $(b, e, h)$, perfis de velocidade $(c, f, i)$, primeiro instante $(a-c)$, segundo instante $(\mathrm{d}-\mathrm{f})$ e terceiro instante $(\mathrm{g}-\mathrm{i})$ 


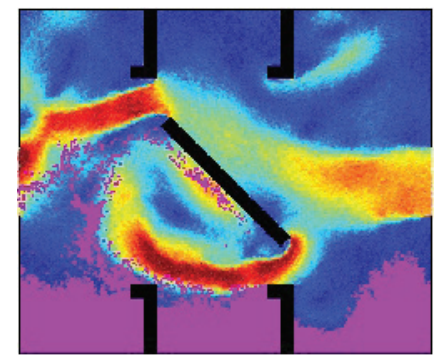

(a)

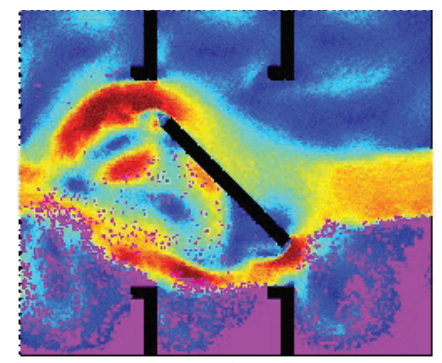

(d)

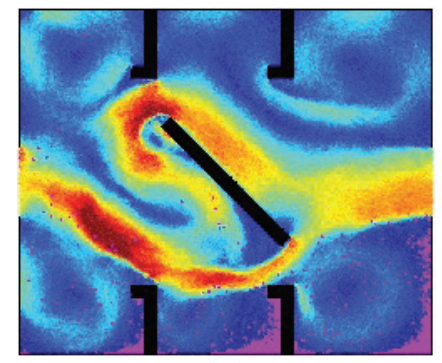

(g)

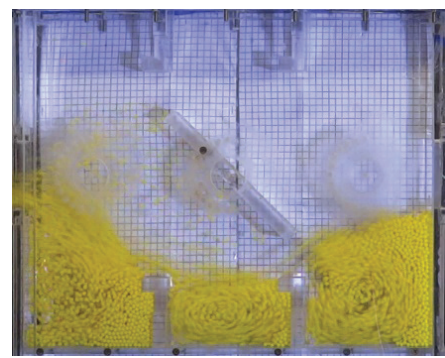

(b)

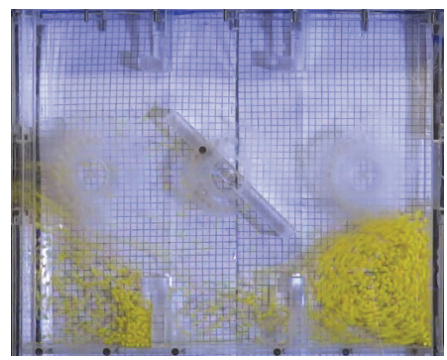

(e)

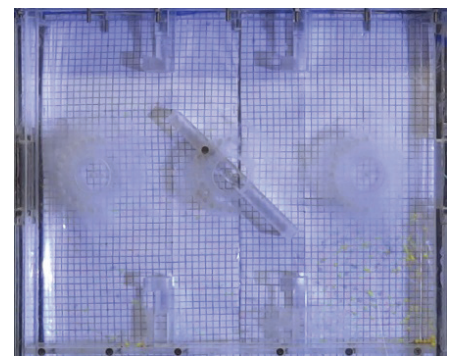

(h)

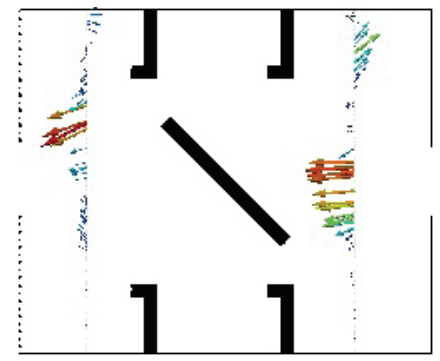

(c)

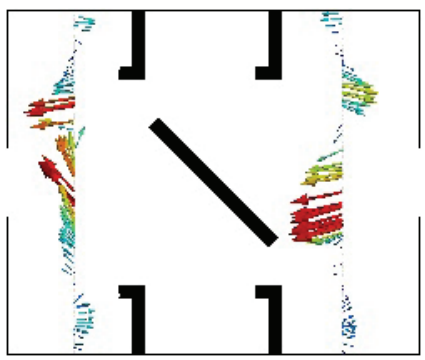

(f)

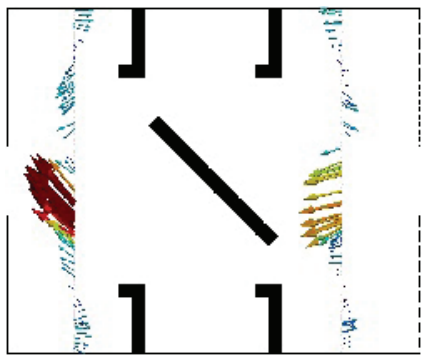

(i)

Velocity (m/s)

0.0

0.3

0.6

0.9

1.2

Figura 44 Comparação dos resultados dos casos AC2: resultado numéricos $(a, d, g)$, resultados experimentais $(b, e, h)$, perfis de velocidade $(c, f, i)$, primeiro instante $(a-c)$, segundo instante $(d-f)$ e terceiro instante $(g-i)$ 


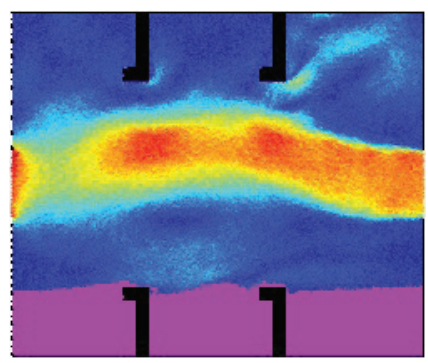

(a)

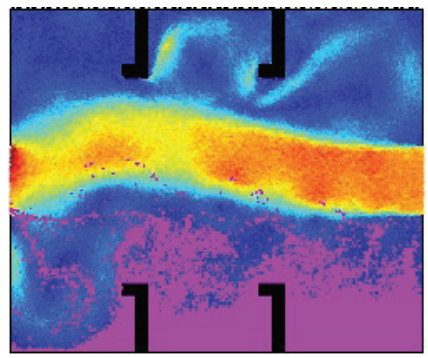

(d)

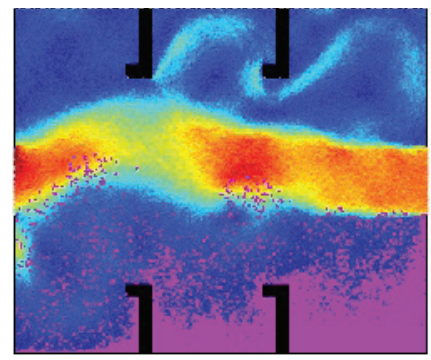

(g)

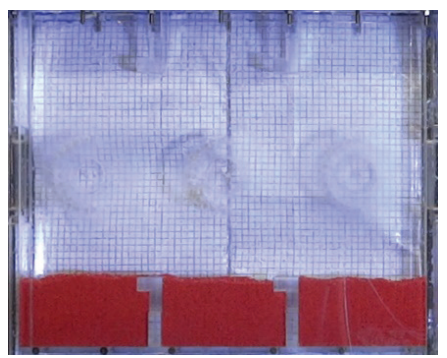

(b)

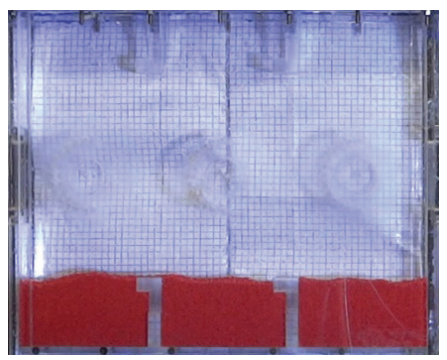

(e)

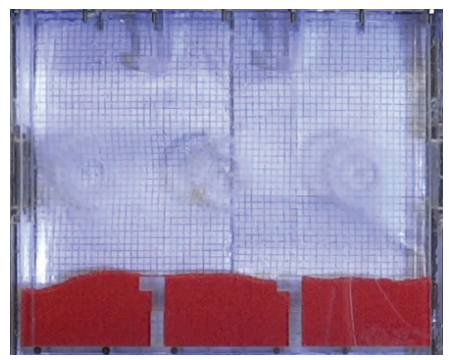

(h)

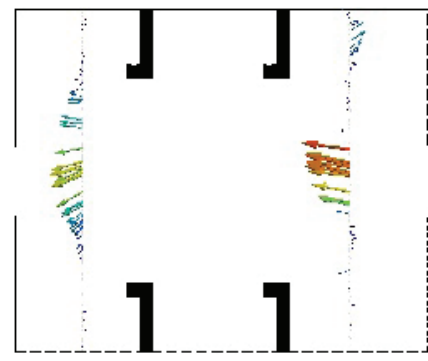

(c)

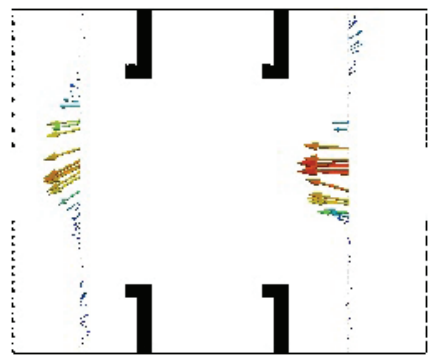

(f)

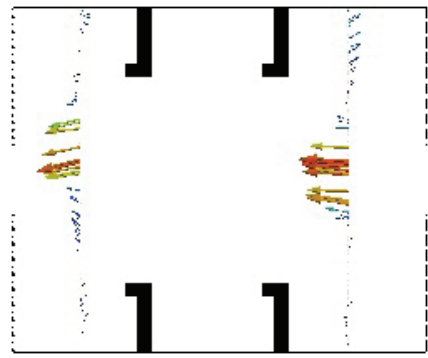

(i)

Velocity $(\mathrm{m} / \mathrm{s})$

0.0

0.3

0.6

0.9

1.2

Figura 45 Comparação dos resultados dos casos MS2: resultado numéricos $(a, d, g)$, resultados experimentais $(b, e, h)$, perfis de velocidade $(c, f, i)$, primeiro instante $(a-c)$, segundo instante $(\mathrm{d}-\mathrm{f})$ e terceiro instante $(\mathrm{g}-\mathrm{i})$ 


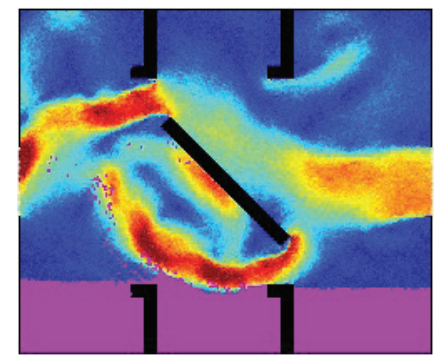

(a)

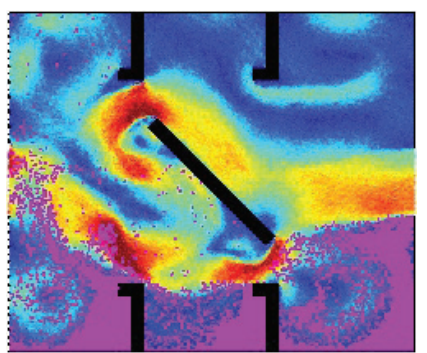

(d)

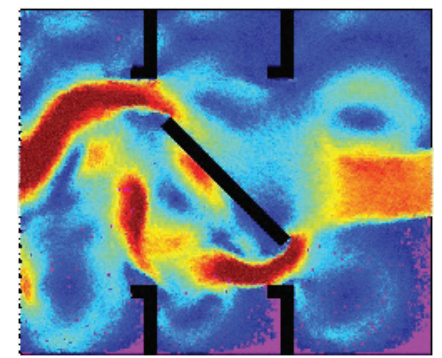

(g)

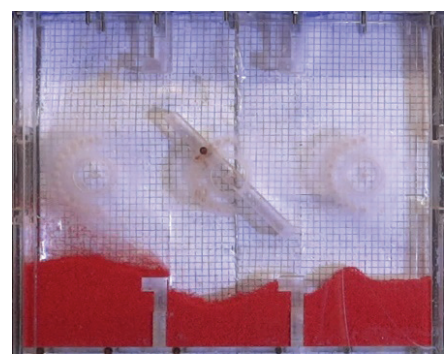

(b)

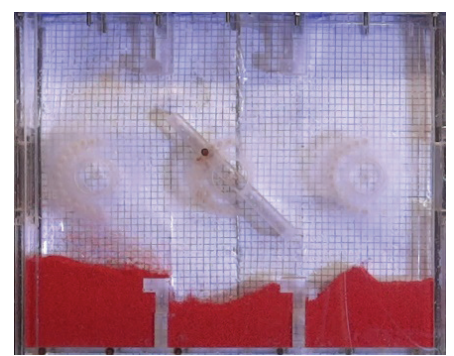

(e)

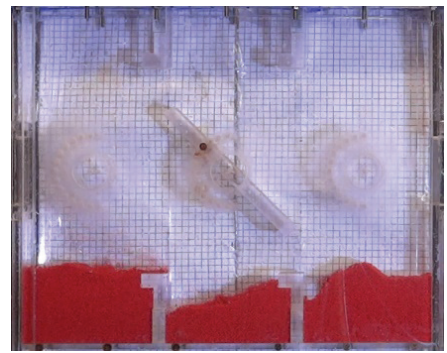

(h)

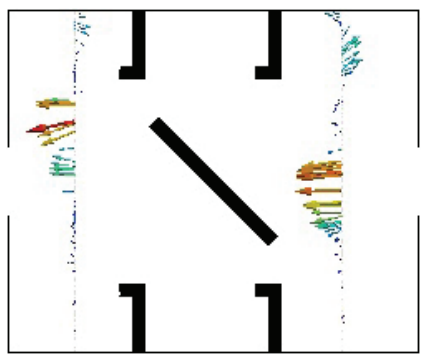

(c)

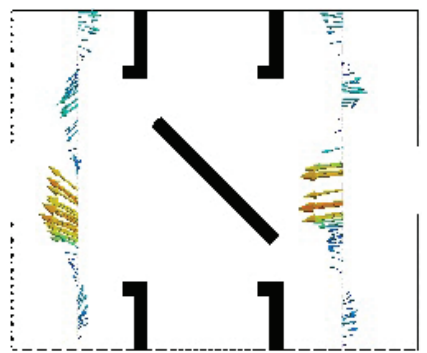

(f)

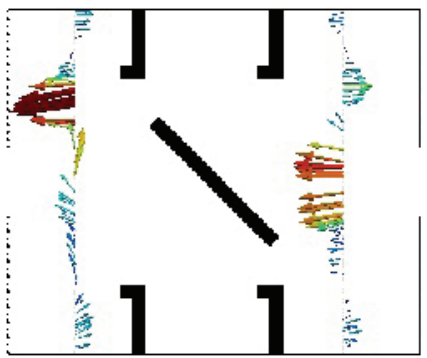

(i)

Velocity $(\mathrm{m} / \mathrm{s})$

0.0

Figura 46 Comparação dos resultados dos casos MC2: resultado numéricos $(a, d, g)$, resultados experimentais $(b, e, h)$, perfis de velocidade $(c, f, i)$, primeiro instante $(a-c)$, segundo instante $(d-f)$ e terceiro instante $(g-i)$

Analisando os resultados apresentados na Figura 47, no primeiro instante, verifica-se a remoção intensiva de sedimentos nos compartimentos do centro e da esquerda, e a formação de um perfil altamente inclinado de sedimentos no compartimento da direita. O resultado numérico permite identificar que a formação de um escoamento de alta velocidade na parte inferior do defletor é responsável pela alta remoção dos sedimentos no compartimento do meio. 
Além disso, em consequência deste escoamento, dois vórtices são formados nos outros dois compartimentos, responsáveis pela remoção dos sedimentos ali acumulados. No segundo instante é possível observar um grande número de partículas em suspensão nos compartimentos da esquerda e da direita, de modo que uma parte significativa dos sedimentos foi removida, no compartimento do meio é possível observar uma resistência maior do sedimento na simulação em relação ao caso experimental. No terceiro instante, é possível verificar quase total remoção de sedimentos no caso experimental, restando poucas partículas em suspensão no compartimento esquerdo. $\mathrm{Na}$ simulação houve permanência um pouco maior de sedimentos nos compartimentos, em específico nas quinas, regiões pouco afetadas pelo vórtice.

É importante ressaltar aqui que, para reduzir o tempo de processamento, as durações de aceleração do experimento e da simulação computacional são diferentes. Enquanto no modelo computacional a duração foi considerada próxima de $2 s$ e imposta a partir do controlador de fluxo, já apresentado em subsecção anterior, no modelo experimental pode ser considerada uma característica resultante das dimensões do modelo, sendo que a duração média observada do período transiente está próxima de $6 \mathrm{~s}$. Disso, pode-se explicar a diferenças observadas nas Figuras 41 - 44. Exemplo desse fato pode ser notado ao se comparar as Figuras 43 (a) e (b), enquanto no experimento são observadas partículas sendo removidas do tanque, na simulação computacional as partículas ainda estão posicionadas próximas da condição inicial.

Enquanto os dois primeiros instantes são influenciados pela duração do período de aceleração inicial, o terceiro instante foi considerado na condição de estabilização do fenômeno de remoção de partículas do tanque. De forma simplificada, o tempo computacional para alcançar esse estágio girava em torno de $30 \mathrm{~s}$ após o fim do período transiente e devido a isso foi adotado como uniforme em todos os casos, possibilitando redução do custo computacional. Em contraparti$\mathrm{da}$, no caso experimental esse instante variava de caso para caso, conforme apresentado na Tabela 10. Alguns casos apresentaram maior tempo, exemplo disso é o caso AS1, que demorou 210,0 s para a estabilização.

A Figura 47 apresenta a evolução da quantidade de partículas de sedimento na simulação ao longo do tempo, com o intuito de verificar as diferenças na remoção de sedimentos para as duas velocidades testadas e para o caso com defletor no interior do tanque de lastro. 

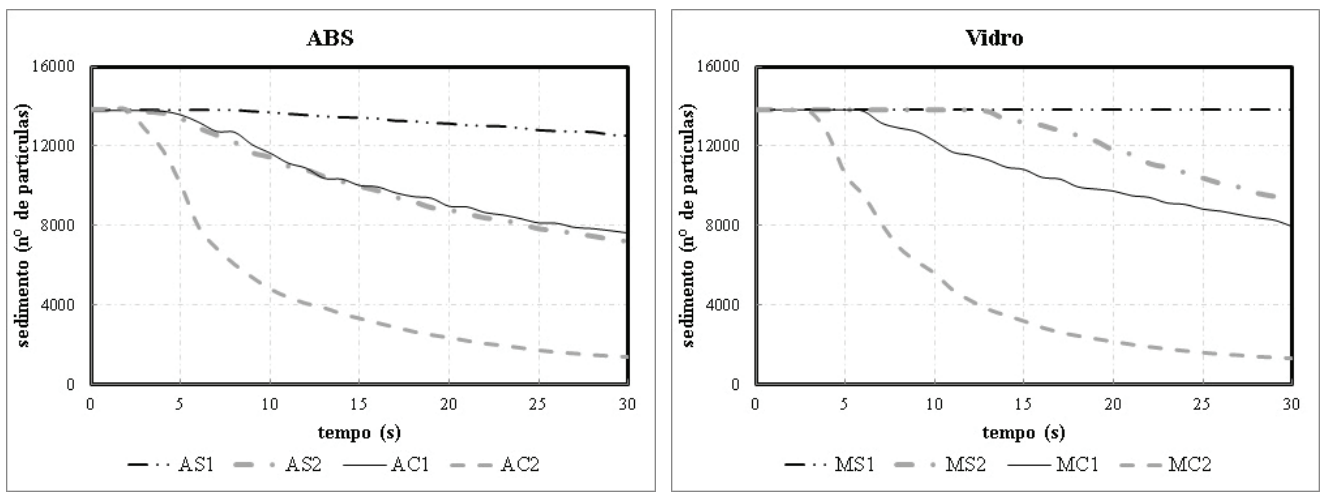

Figura 47 Quantidade de partículas de sedimento ao longo do tempo

Tabela 10 Comparação do tempo

\begin{tabular}{|c|c|c|c|c|c|c|}
\hline \multirow{2}{*}{ Caso } & \multicolumn{2}{|c|}{ Primeiro instante (s) } & \multicolumn{2}{c|}{ Segundo instante (s) } & \multicolumn{2}{|c|}{ Terceiro instante (s) } \\
\cline { 2 - 7 } & MPS & EXP. & MPS & EXP. & MPS & EXP. \\
\hline AS1 & 2,00 & 6,00 & 22,00 & 29,47 & 32,00 & 210,00 \\
\hline AS2 & 2,00 & 8,00 & 12,00 & 17,17 & 32,00 & 93,00 \\
\hline AC1 & 2,00 & 7,00 & 22,00 & 28,84 & 32,00 & 73,00 \\
\hline AC2 & 2,00 & 6,00 & 12,00 & 14,95 & 32,00 & 39,00 \\
\hline MS1 & 2,00 & 5,00 & 22,00 & 25,85 & 32,00 & 32,00 \\
\hline MS2 & 2,00 & 7,00 & 12,00 & 15,69 & 32,00 & 54,00 \\
\hline MC1 & 2,00 & 9,00 & 22,00 & 35,20 & 32,00 & 80,00 \\
\hline MC2 & 2,00 & 8,00 & 12,00 & 17,69 & 32,00 & 77,00 \\
\hline
\end{tabular}

\subsection{CONSIDERAÇÕES FINAIS}

Neste trabalho abordagens experimental e numérica são adotadas para investigar os mecanismos de remoção de sedimento nos tanques de duplo fundo. Por simplicidade, experimentos físicos foram conduzidos, onde o sedimento é aproximado por pequenas esferas de material homogêneo. Numericamente o sedimento e a água foram modelados como fluídos homogêneos, newtonianos, imiscíveis e com diferente densidade e o método Moving Particle Semi-Implicit (MPS), baseado em descrição lagrangeana e discretização do domínio em partículas, foi adotado para melhor capturar a complexa interface entre fluídos. A fim de estimular o processo de remoção, introdução de um defletor no tanque foi considerado, e os seus efeitos analisados. 
Como resultado, para o sedimento leve (ABS) é possível observar que com velocidade de escoamento relativamente baixa, em torno de 0,45 m/s, a remoção de sedimentos é ineficaz em um tanque de geometria complexa usual. Quando a velocidade do escoamento é duas vezes maior, ocorre uma remoção mais eficaz de sedimentos, retirando aproximadamente $50 \%$ do material depositado.

A adição do defletor facilita a remoção dos sedimentos ao criar vórtices de maior intensidade nos compartimentos do tanque, de modo que com baixa velocidade de escoamento obtém-se um desempenho similar a alta velocidade de escoamento sem defletor. A maior eficácia pode se alcançada pela combinação de alta velocidade de escoamento e presença do defletor, situação em que os vórtices têm energia suficiente para remoção quase completa dos sedimentos.

Os resultados experimentais e numéricos mostram que os sedimentos de maior densidade (vidro) dificultam a remoção. No entanto, enquanto existe boa aderência dos resultados numéricos e experimentais ao se considerar o sedimento de baixa densidade, a diferença entre os resultados numéricos e experimentais aumenta para o caso do sedimento com maior densidade. Uma explicação para isso é a modelagem computacional simplificada que não leva em conta o atrito estático entre as esferas, que é mais relevante no caso do sedimento de maior densidade, impedindo a suspensão e a remoção dos sedimentos.

Outro fator que deve ter influenciado é a diferença dos diâmetros dos sedimentos utilizados nos ensaios experimentais, que no caso de ABS é próximo de $6 \mathrm{~mm}$ e para o vidro é aproximadamente de $2 \mathrm{~mm}$. O maior diâmetro das partículas resulta em interstícios maiores para o ABS, o que não é observado no caso dos sedimentos de vidro, onde as forças coesivas tendem a ser maiores.

No entanto, apesar das simplificações adotadas na modelagem do sedimento, foi possível observar uma boa concordância da hidrodinâmica entre os resultados numéricos e os resultados experimentais principalmente no caso de alta vazão com ABS. Além disso, a modelagem computacional permitiu reproduzir o comportamento geral do processo e, para velocidades maiores e com sedimentos de menor densidade, a modelagem utilizada é eficaz.

\subsection{AGRADECIMENTOS}

Os autores desse estudo agradecem à FINEP pelo financiamento ao projeto "Desenvolvimento de sistemas de monitoramento da água de lastro com avaliação de forçantes hidrodinâmicas, operacionais e biológicas”. 


\subsection{REFERÊNCIAS}

Bellezi, C. A., Cheng, L.-Y, Nishimoto, K, 2013. Flow conditioning modelling and application to particle method, III International Conference on Particle-based Methods - Fundamentals and Applications PARTICLES 2013.

Koshizuka, S., Oka, Y., 1996. Moving-Particle Semi-Implicit method for fragmentation of incompressible fluid. Nuclear Science and Engineering, vol. 123, p. 421-434. 